\title{
High-resolution mass spectrometry and velocity map imaging for ultrafast electron dynamics in complex biomolecules
}

\author{
Erik P. Månsson ${ }^{1, *}$, Vincent Wanie ${ }^{2,3}$, Mara Galli $^{2,4}$, Mattea C. Castrovilli ${ }^{5}$, Fabio \\ Frassetto $^{6}$, Luca Poletto $^{6}$, Mauro Nisoli ${ }^{2,4}$, and Francesca Calegari ${ }^{1,2}$ \\ ${ }^{1}$ Center for Free-Electron Laser Science, DESY, 22607 Hamburg, Germany \\ ${ }^{2}$ Institute for Photonics and Nanotechnologies, National Research Council, 20133 Milano, Italy \\ ${ }^{3}$ Institut National de la Recherce Scientifique, J3X1S2, Varennes (Qc), Canada \\ ${ }^{4}$ Department of Physics, Politecnico di Milano, 20133 Milano, Italy \\ ${ }^{5}$ Institute for the Structure of Matter, National Research Council, Monterotondo, Italy \\ ${ }^{6}$ Institute for Photonics and Nanotechnologies, National Research Council, 35131 Padova, Italy
}

\begin{abstract}
We present a design combining a velocity map imaging electron spectrometer with a reflectron mass spectrometer. Since the two spectrometer sides have different intrinsic requirements for the electric field in the central region, a large number of electrodes and a reflectrongeometry of the mass spectrometer were employed to achieve simultaneous high resolutions. Together with femtosecond and attosecond pump-probe methods it will enable studies of ultrafast dynamics in large molecular systems.
\end{abstract}

\section{Introduction}

Pump-probe methods with few-femtosecond laser pulses are often used to study the motion of nuclei during photochemical reactions. With attosecond pulses in the extreme ultraviolet (XUV) spectral range the electron dynamics underlying charge transfer and the breaking/formation of chemical bonds can be followed on its natural time scale [1,2]. This research field is progressing towards more complex samples such as surfaces, nanoparticles and biologically relevant molecules. We recently studied some of the nucleosides (RNA/DNA constituents with masses of 227-283 u) evaporated in vacuum, by initiating dynamics with an XUV attosecond pump pulse, probing it with a near-IR pulse and resolving the ionic dissociation fragments by mass [3]. Our ongoing development of sub-2 fs UV-pulses will also allow dynamics in neutral molecules to be studied in the future.

To distinguish isotopes or protonation in biomolecules and to extend our work towards electron dynamics in short RNA-chains or peptides it is necessary to work with higher mass resolution. In the present work we describe a new instrument that combines a reflectrontype [4,5] mass spectrometer with a velocity map imaging spectrometer on the opposite side (Fig. 1). While molecular states are not generally resolved in the continuous electron spectra from photoionization by an isolated attosecond pulse, the use of a few-femtosecond

* Corresponding author: erik.maansson@desy.de 
pulse in the UV will provide simpler electron spectra. Electronic relaxation involving autoionization will also leave a fingerprint of electrons with specific kinetic energies.

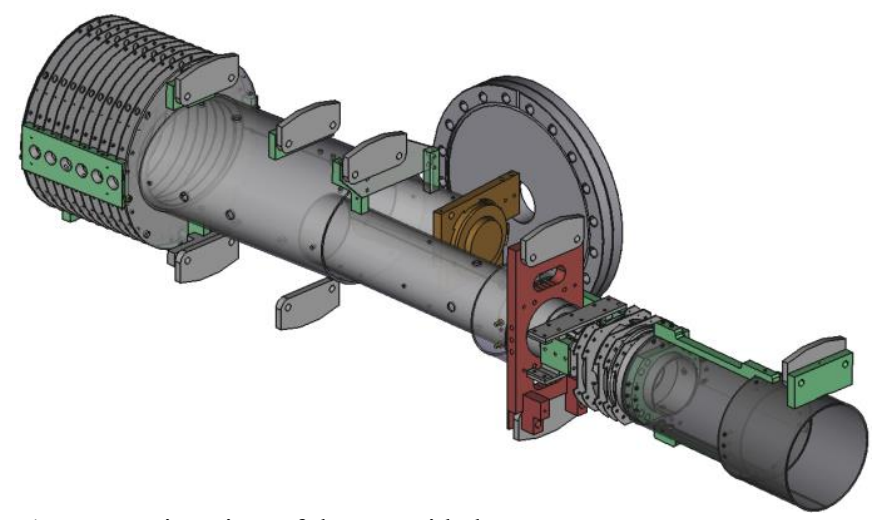

Fig. 1. Perspective view of the two-sided spectrometer.

\section{Mass spectrometer design}

A time-of-flight mass spectrometer essentially consists of an electric field that extracts the ions and a drift tube that enlarges the flight time before reaching the detector. The massresolution can be analysed as the differential effect on the time of flight due to spread in the ions' starting position and due to spread in their initial kinetic energy [6]:

- The range of starting positions is mainly given by the intersection of the optical beam width the sample density (ideally a molecular beam). An ion starting closer to the extracting electrode needs less time to reach the drift tube (which we can call negative dispersion) but it also starts at a lower electrical potential and will therefore travel more slowly in the drift tube (positive dispersion). The positive and negative dispersion contributions can be made to cancel each other by choosing a specific length ratio between the extracting and drift regions $[5,6]$. For cold parent ions this has a large effect on the mass resolution, improving it from a baseline around $100 \mathrm{u}$ to past $10^{4} \mathrm{u}$ in specialized instruments $[5,7,8]$. To approach this from a typical velocity map imaging spectrometer requires a longer extraction region, shorter drift tube, or voltages that keep increasing within the drift tube (which then becomes another accelerating region).

- Kinetic energy is always present in the form of thermal energy of the sample, although this can be reduced by molecular beam techniques for the gas-expansion into the vacuum chamber and by an atomic buffer gas. Considerably higher kinetic energy is carried by dissociation fragments. To reduce the time-of-flight-blurring effect of initial kinetic energy, the extraction-voltage can be increased and the drift tube made longer.

Since a velocity map imaging spectrometer, primarily for electrons, is desired on the opposite side of the mass spectrometer the shared extraction region is strongly constrained in size and electrical voltage. The microchannel-plate detectors at both sides should be kept within a handful of kilovolts from ground. From the above analysis of time-of-flight dispersion we see that this leaves us with conflicting demands for the drift tube length: making it shorter to handle the position-spread of the ions or longer to handle their kinetic spread. The solution is to install a reflectron $[4,5,7]$ where ions are decelerated and reversed, as illustrated on the left side of Fig. 2. The reflectron contributes an adjustable amount of the so-called negative dispersion, making a longer drift tube acceptable. 


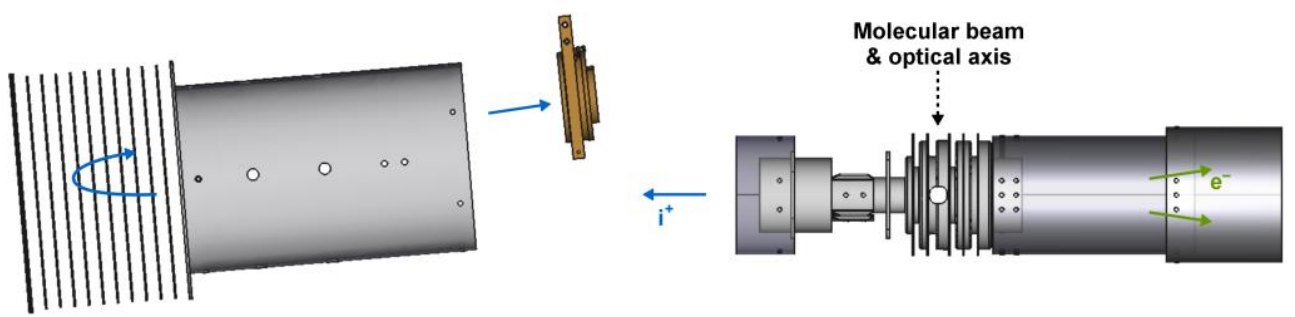

Fig. 2. Plane-view of most of the electrodes. Arrows sketch the trajectories for cations on the mass spectrometer side (left) and electrons on the velocity map imaging side (right). The total length of the spectrometer is just less than $1 \mathrm{~m}$.

Simulations of the chosen design, while requiring electron imaging up to $30 \mathrm{eV}$ with better than $1 \mathrm{eV}$ energy resolution, suggest that the simultaneous mass resolution approaches $10^{4} \mathrm{u}$ in the case of parent ions from a cold molecular beam, $1500 \mathrm{u}$ at room temperature and $150 \mathrm{u}$ for $2-\mathrm{eV}$ dissociation fragments. This represents an order of magnitude improvement from our previous, one-sided, spectrometer.

\section{Conclusions}

The new spectrometer will allow resolving much heavier ions than hitherto studied in attosecond laboratories and give simultaneous access to the spectral and angular distribution of electrons, so that the covariance [9] of electron spectra and mass spectra can give precious information about the states involved in molecular photoreactions.

\section{References}

1. F. Calegari, D. Ayuso, A. Trabattoni, L. Belshaw, S. De Camillis, S. Anumula, F. Frassetto, L. Poletto, A. Palacios, P. Decleva, J. B. Greenwood, F. Martín, and M. Nisoli. Science 346, 336 (2014).

2. L. Belshaw, F. Calegari, M. J. Duffy, A. Trabattoni, L. Poletto, M. Nisoli, and J. B. Greenwood. J. Phys. Chem. Lett. 3, 3751 (2012).

3. E. P. Månsson, S. De Camillis, M. C. Castrovilli, M. Galli, M. Nisoli, F. Calegari, and J. B. Greenwood. Phys. Chem. Chem. Phys. 19, 19815 (2017).

4. S. Scherer, K. Altwegg, H. Balsiger, J. Fischer, A. Jäckel, A. Korth, M. Mildner, D. Piazza, H. Reme, and P. Wurz. International Journal of Mass Spectrometry 251, 73 (2006).

5. F. Chandezon, E. Huber, and C. Ristori. Rev. Sci. Instrum. 65, 3344 (1994).

6. E. P. Månsson, S. L. Sorensen, C. L. Arnold, D. Kroon, D. Guénot, T. Fordell, F. Lépine, P. Johnsson, A. L'Huillier, and M. Gisselbrecht. Rev. Sci. Instrum. 85, 123304 (2014).

7. J. Lee, H. Chen, T. Liu, C. E. Berkman, and P. T. A. Reilly. Anal. Chem. 83, 9406 (2011).

8. G. R. Hilton and J. L. P. Benesch. J. Royal Socicety Interface 9, 801 (2012).

9. O. Kornilov, M. Eckstein, M. Rosenblatt, C. P. Schulz, K. Motomura, A. Rouzée, J. Klei, L. Foucar, M. Siano, A. Lübcke. J. Phys. B 46, 164028 (2013). 